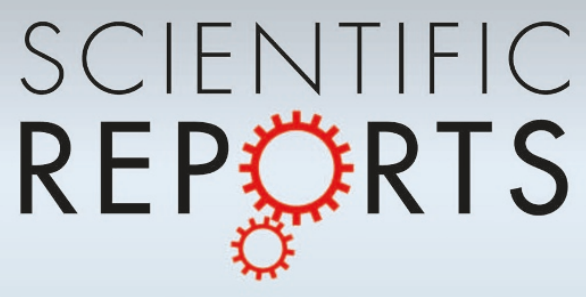

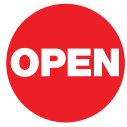

SUBJECT AREAS:

MATERIALS SCIENCE

APPLIED PHYSICS

MECHANICAL ENGINEERING

MINERALOGY

Received

24 September 2012

Accepted

30 October 2012

Published

19 November 2012

Correspondence and requests for materials should be addressed to X.H.Y. (xiaohui@lanl. gov) or Y.S.Z. (yusheng.zhao@unlv.

edu)

\title{
Constitutive Law and Flow Mechanism in Diamond Deformation
}

\author{
Xiaohui Yu' ${ }^{1}$ Paul Raterron' ${ }^{2}$, Jianzhong Zhang ${ }^{1}$, Zhijun Lin' ${ }^{1}$ Liping Wang ${ }^{3} \&$ Yusheng Zhao ${ }^{1,3}$ \\ 'LANSCE Lujan Center, Los Alamos National Laboratory, Los Alamos, New Mexico 87545, USA, ${ }^{2}$ Unité Matériaux et \\ Transformations, CNRS 8207, Bât C6, Université Lille 1, F-59655 Villeneuve d'Ascq Cedex, France, ${ }^{3}$ HiPSEC and Department of \\ Physics and Astronomy, University of Nevada, Las Vegas, Nevada 89154, USA.
}

Constitutive laws and crystal plasticity in diamond deformation have been the subjects of substantial interest since synthetic diamond was made in 1950's. To date, however, little is known quantitatively regarding its brittle-ductile properties and yield strength at high temperatures. Here we report, for the first time, the strain-stress constitutive relations and experimental demonstration of deformation mechanisms under confined high pressure. The deformation at room temperature is essentially brittle, cataclastic, and mostly accommodated by fracturing on $\{111\}$ plane with no plastic yielding at uniaxial strains up to $15 \%$. At elevated temperatures of $1000^{\circ} \mathrm{C}$ and $1200^{\circ} \mathrm{C}$ diamond crystals exhibit significant ductile flow with corresponding yield strength of 7.9 and $6.3 \mathrm{GPa}$, indicating that diamond starts to weaken when temperature is over $1000^{\circ} \mathrm{C}$. At high temperature the plastic deformation and ductile flow is meditated by the $<110>\{111\}$ dislocation glide and a very active $\{111\}$ micro-twinning.

F or centuries it has been known that diamond is brittle and can easily cleave when crafting on a certain crystallographic plane, the $\{111\}$ plane as we know it now, which also has the lowest cleavage energy ${ }^{1,2}$. However, as the hardest material known to mankind, diamond is experimentally difficult to deform, particularly at room temperature. The current knowledge of plastic deformation in diamond is mainly rested on first principles calculations and on experiments under uncontrolled stress conditions. Theoretically, the strain-stress relations are derived for diamond based on simulations of perfect crystals at zero Kelvin ${ }^{2-5}$. Typically, these calculations do not take into account the contributions from crystalline defects such as dislocations $^{6}$ and twinning ${ }^{7}$, which are the major factors affecting materials' strength. Experimental strength measurements for diamond include indentation, bending, and grinding ${ }^{1,8,9}$. In high-pressure experiments in diamond anvil cells, the strength of diamond can also be estimated by optical absorption ${ }^{10}$, luminescence ${ }^{11}$, Raman spectroscopy ${ }^{12}$, and X-ray imaging ${ }^{13}$, but the results are often inconsistent and cannot directly be converted to compressional or tensile strength. Another method for the strength measurement was based on diffraction peak width analysis of polycrystalline diamond under high pressure $(P)$ and high temperature $(T)$ condition $^{14}$. The derived strength, however, is commonly referred to as microscopic strength, which is primarily associated with the concentrated local-/microscopic- strains at the contact points of crystal grains. Because all these experimental methodologies cannot quantify the stress applied during deformation, the classic strain-stress relation, which is fundamentally important to the plasticity of diamond, has yet to be established under both room and elevated temperatures.

We studied deformation behavior of polycrystalline diamond at room temperature, $1000^{\circ} \mathrm{C}$, and $1200^{\circ} \mathrm{C}$, near confined pressures of $3.5 \mathrm{GPa}$. The uniaxial high $P$ - $T$ deformation experiments were carried out in a deformation-DIA apparatus (D-DIA) interfaced with synchrotron $\mathrm{x}$-ray diffraction and radiography ${ }^{15}$. At any given deformation state, the macroscopic differential stress is derived from elastic distortion in a crystalline lattice and the total axial strain is measured by $\mathrm{x}$-ray imaging of the bulk sample. We also derived the microscopic strain based on diffraction profile analysis. To gain insights into the deformation mechanisms, microstructures of the deformed samples were systematically characterized by optical microscopy, scanning electron microscopy (SEM), and transmitted electron microscopy (TEM). Experimental details are described in the Methods Section.

\section{Results}

Microscopic and macroscopic strain-stress measurements. Figs. 1a and $1 \mathrm{~b}$ show microscopic and macroscopic strain-stress curves for diamond deformed at a confining pressure around $3.5 \mathrm{GPa}$ and room temperature. As expected, both curves show slow stress build up compared to classic behaviors in metals. Markedly, there is a stress plateau in microscopic stress (Fig. 1a) or stepped variation in macroscopic stress (Fig. 1b) between $1 \%$ and 

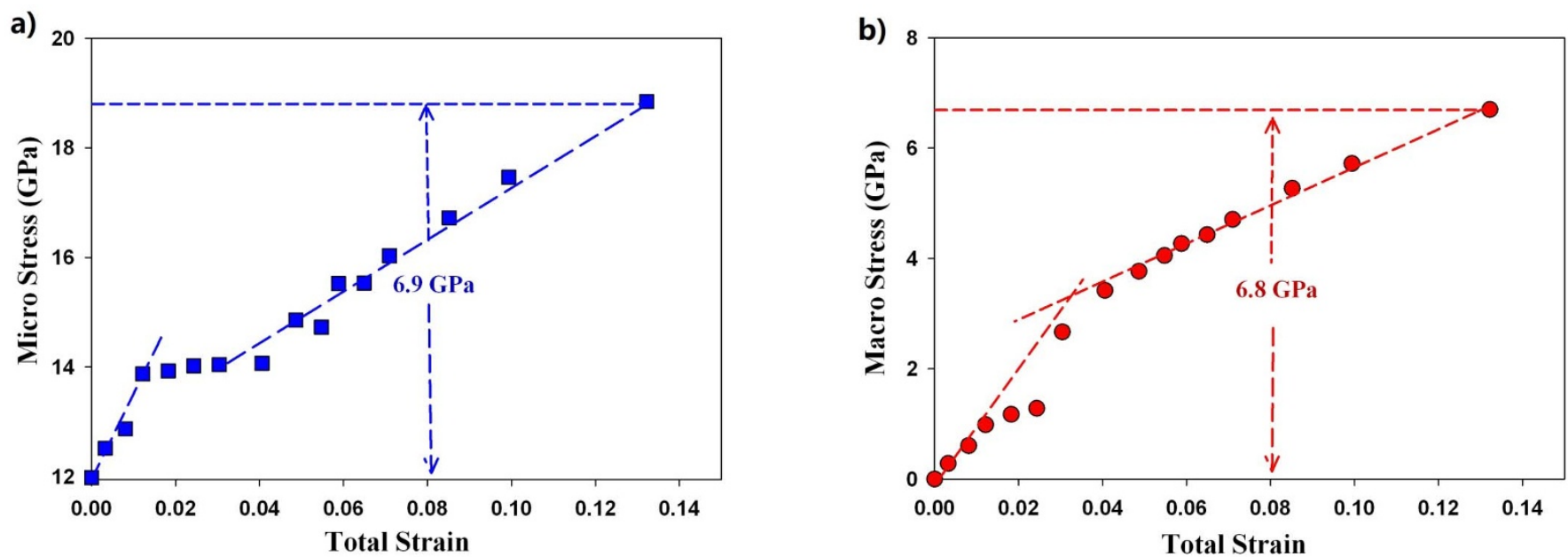

c)

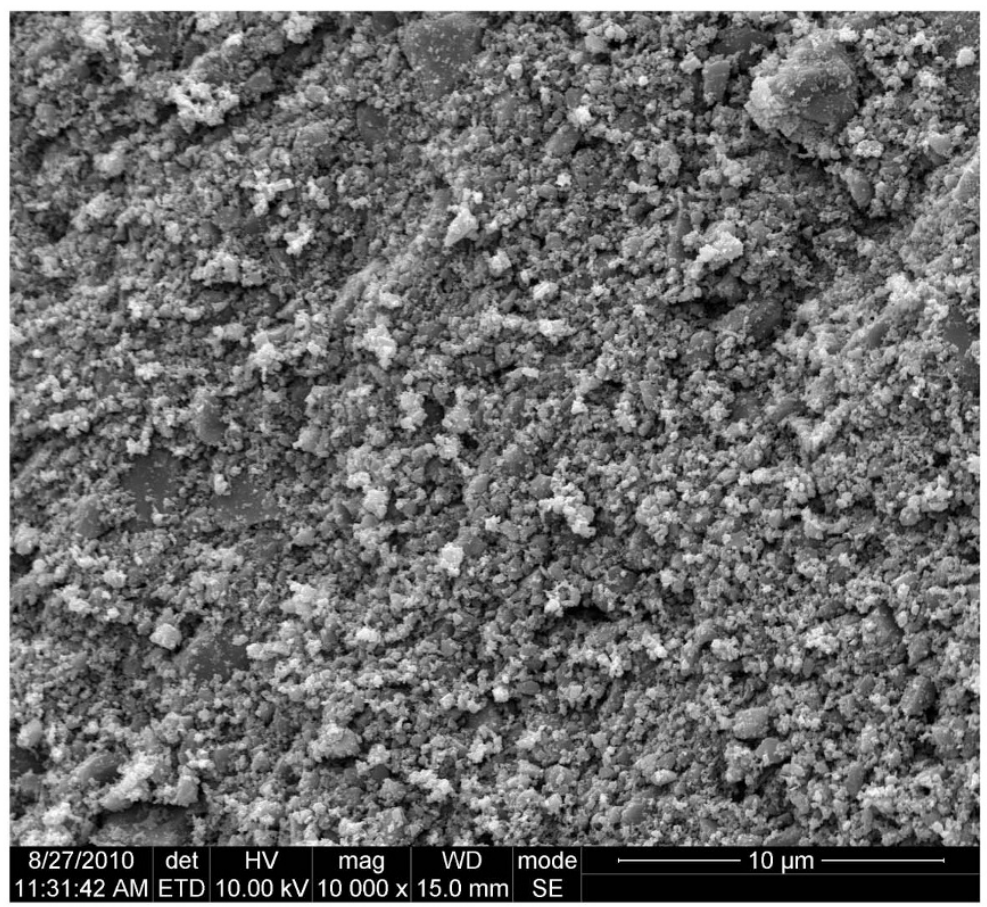

Figure $1 \mid$ (a) Microscopic and (b) macroscopic stress as a function of total strain for polycrystalline diamond deformed at room temperature and a confining pressure of $3.5 \mathrm{GPa}$; (c) SEM image of the recovered sample.

$4 \%$ total strains. Such unusual "flow" indicates a stress relaxation or strain adjusting state. Beyond the discontinuities, the stress continues to increase but at slower paces than initially observed; no plastic yielding, however, is observed at total axial strains up to $15 \%$. After the deformation experiment, the recovered sample was characterized by SEM to gain insight into microstructural features, which are shown in Fig. 1c. Evidently, diamond crystals with starting grain sizes of 4 to 6 microns have been fractured to submicron or even nano scale. These combined observations indicate that the deformation of polycrystalline diamond at room temperature is elastic in nature, and the observed stress-relaxation/strain-adjusting demonstrates brittle fracture and cataclastic flow in polycrystalline compacts.

Another feature of Figs. 1a and $1 \mathrm{~b}$ is that the microscopic and macroscopic stresses show similar variation with total strain up to $15 \%$. In particular, the built-up microscopic stress of 6.9 GPa derived from the peak width analysis is essentially identical to the macroscopic stress of $6.8 \mathrm{GPa}$ determined from the diffraction lattice strain. It indicates that, at room temperature, the concentrated local grain-to-grain contact stress is on average equal to the bulk differential stress applied to the entire sample. During the deformation as differential stress is built up, the brittle fracture behavior dominates the entire deformation process through crystal cleavage on $\{111\}$ plane. This is particularly true at the cataclastic flow stage as fractured grains release part of the differential stress and the rolling/ tumbling of diamond grains can continue to support the flow stress. It is also noted that the microscopic stress is much higher in absolute magnitude than the macroscopic stress. This is because, prior to the uniaxial deformation at $3.5 \mathrm{GPa}$, micro-stresses are already highly concentrated at gain-to-grain contact points during initial pressurization, which would in turn facilitate cleavage of diamond grains, as revealed by TEM micrographs in the TEM section. 
The strain-stress curves at $1000^{\circ} \mathrm{C}$ are shown in Figs. $2 \mathrm{a}$ and $2 \mathrm{~b}$. Different from the room-temperature deformation, diamond crystals reach the ultimate yield with a flow stress of $7.9 \mathrm{GPa}$ at a total strain of around $11 \%$. Notably, the stress plateau or step revealed in the room temperature strain-stress curves is not observed, indicating that the deformation mechanism has changed from brittle fracture mode at room-temperature to ductile mode at $1000^{\circ} \mathrm{C}$. Thus, the deformation process at this high temperature should be dominated by dislocation-mediated plasticity instead of crystal cleavage. The brittle and cataclastic component may still be active at $1000^{\circ} \mathrm{C}$ but its contribution to diamond deformation is insignificant based on the observations discussed below. Similar to room-temperature measurements, the microscopic and macroscopic strain-stress curves show same trends of variation during the deformation, indicating that even at $1000^{\circ} \mathrm{C}$ diamond crystal grains are sufficiently strong to support additional local concentrated stresses.

To further understand the deformation behavior of polycrystalline diamond at high temperature, we carried out an additional deformation experiment at $1200^{\circ} \mathrm{C}$. The derived strain-stress curves are shown in Figs. 3a and 3b. Strikingly, no micro-stress build up is observed during the entire course of bulk deformation. This, when compared with the observations of Figs. $2 \mathrm{a}$ and $2 \mathrm{~b}$, indicates that there exists a threshold temperature between $1000^{\circ} \mathrm{C}$ and $1200^{\circ} \mathrm{C}$ above which diamond crystals become sufficiently weakened to support additional local concentrated differential stresses. This is in good agreement with an early work by Weidner et al, who showed that differential microscopic stress of diamond under tri-axial loading starts decreasing rapidly above $1100^{\circ} \mathrm{C}^{14}$. However, the macroscopic strain-stress can still be built up during the bulk sample deformation at $1200^{\circ} \mathrm{C}$ as long as the stress is below the yield strength of diamond. The SEM characterization of the recovered sample, Fig $3 c$, shows that there is no obvious grain size reduction after the deformation, indicating that at $1200^{\circ} \mathrm{C}$ brittle fracture or cataclastic flow does not make significant contributions to the diamond deformation. Instead, the deformation should be dominated by dislocation mediated plasticity.

TEM characterization and deformation mechanism at room temperature. Although diamond has the highest hardness and shear modulus owing to $\mathrm{sp}^{3}$ hybridization and directional bonding of carbon atoms, diamond crystals cleave quite easily on a certain crystallographic plane when undergoing uni-axial deformation at room temperature. According to first principles calculations of Telling et $\mathrm{al}^{2}$, the energy ratio required to cleave the $\{100\},\{110\}$,

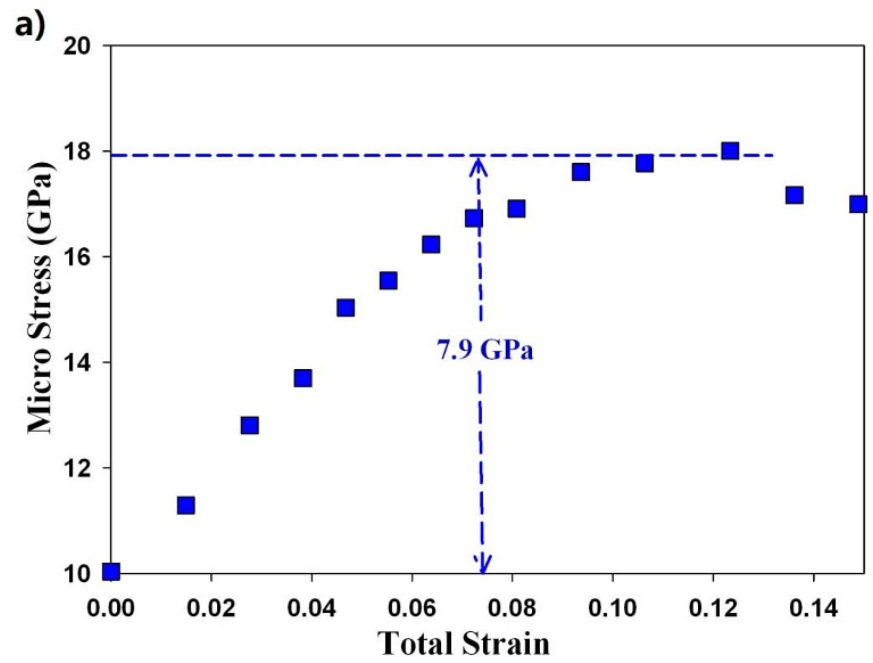

and $\{111\}$ planes in diamond crystals is approximately $7: 2.8: 1$, indicating that the $\{111\}$ plane can more readily be fractured than the other planes ${ }^{2}$. While this has commonly been observed in the processes of diamond cutting and shaping ${ }^{1}$, microstructure studies of indented diamonds revealed both $\{111\}^{1,16}$ and $\{001\}^{17}$ cleavage planes. To date, the exact fracture mechanism has yet been elucidated by controlled deformation experiments. In order to shed light on the cleavage planes in brittle fracturing, we carried out TEM characterizations of the sample recovered from roomtemperature deformation (DIA300). As revealed by TEM micrograph (Fig. 4a), large fractured crystals of a few microns in size, mostly free of intra-crystalline defects such as dislocations, are surrounded by smaller diamond crystals. Within the large grains, micro-fractures mostly occur on $\{111\}$ cleavage planes: either as long straight cleavages or curved fractures consisting of numerous nano-steps along $\{111\}$ cleavage planes (Fig. 4b). In the small-grain areas, grains or sub-grain domains as small as $10 \mathrm{~nm}$ are visible in bright-field (BF) imaging mode. The use of a 1.5- $\mu \mathrm{m}$ large selectedarea (SA) aperture also produces spotty Debye rings in diffraction mode (Fig. 4a). In these areas the grain size ranges typically from $10 \mathrm{~nm}$ to a few $100 \mathrm{~nm}$, although grains less than $10 \mathrm{~nm}$ (invisible in micrographs) may also be present. The very few straight dislocations observed in the deformed sample suggest that they are not glissile and are likely growth defects. These microstructures attest that the deformation at room temperature was essentially brittle and the observed flow was cataclastic, and mostly accommodated by fracturing (cleavage) on $\{111\}$ plane which is the weakest crystallographic plane in diamond ${ }^{2}$. Clearly, our results do not support the $\{001\}$ fracture mode revealed in the indentation experiment ${ }^{17}$.

TEM characterization and deformation mechanism at $1200{ }^{\circ} \mathrm{C}$. At the TEM scale, the recovered diamond grains exhibit extremely high densities of $\langle 110\rangle$ dislocations, the classic dislocations in diamond, and planar defects on $\{111\}$ planes (Fig. 5a). The dislocations are glissile, as revealed by their curvatures, and exhibit numerous entanglements which render it difficult to characterize their glide planes. The planar defects are mostly micro-twins, as revealed by their classical moiré fringes in dark-field condition (Fig. 5b) and by the splitting of reflections in diffraction micrographs obtained with $<110>$ zone axis (Fig. $5 c$ ). The density of $\{111\}$ microtwins is high in the $1200^{\circ} \mathrm{C}$ deformed sample which explains the anomalously visible and strong 200 diffraction spots in diffraction patterns (e.g., Fig. 5c), as was previously reported for electron diffraction patterns arising from twinned diamonds and diamond films ${ }^{18,19}$. These

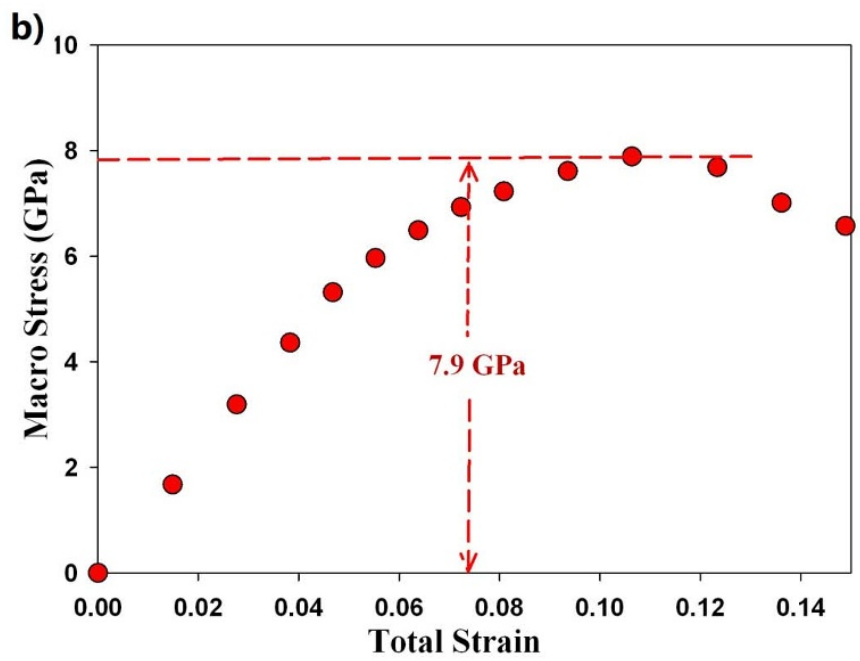

Figure $2 \mid$ (a) Microscopic strain-stress curve and b) macroscopic strain-stress curve for polycrystalline diamond deformed at $1000^{\circ} \mathrm{C}$ and $3.5 \mathrm{GPa}$. 

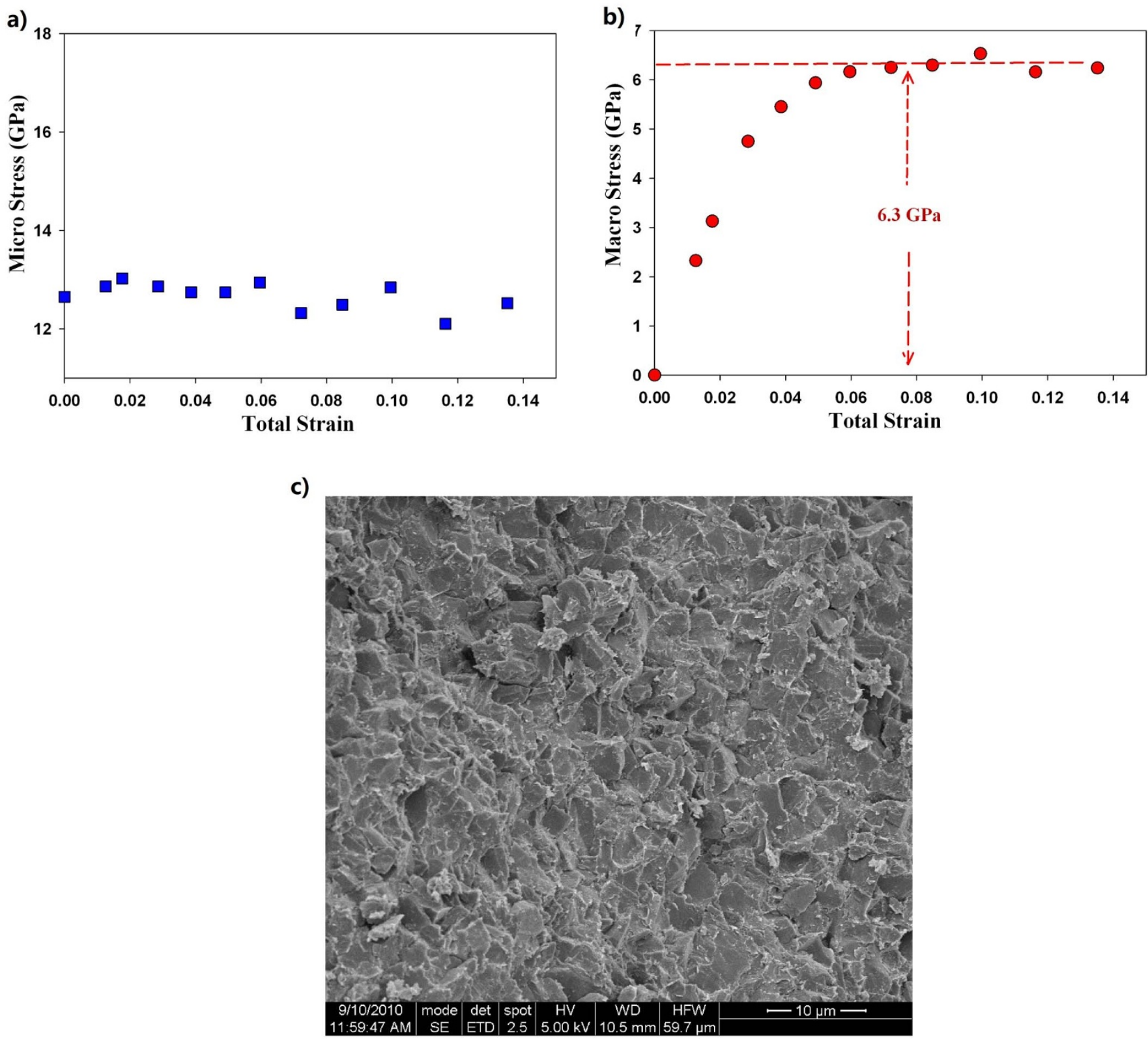

Figure 3 (a) Microscopic strain-stress curve and (b) macroscopic strain-stress curve for polycrystalline diamond deformed at $1200^{\circ} \mathrm{C}$ and $\sim 4.0 \mathrm{GPa}$. Because the deformation experiment was conducted at a higher temperature of $1200^{\circ} \mathrm{C}$ a higher pressure was applied to prevent potential graphitization of diamond; (c) SEM image of the recovered sample.

microstructures show that plastic deformation in diamond is accommodated by $<110>\{111\}$ dislocation glide and a very active $\{111\}$ microtwinning.

\section{Discussion}

Experimental determination of constitutive laws and flow mechanisms in diamond deformation has been a standing problem since synthetic diamond was made in 1950's. Yield strength is in this regard an important property because it defines the onset of plastic deformation and viscous flow. Although the strongest material, diamond crystals cleave on $\{111\}$ planes at strains as small as $1 \%$ at room temperature, which is in line with the common observations of low fracture toughness in single-crystal diamond. At elevated temperatures, diamond starts weakening at temperatures as low as $1000^{\circ} \mathrm{C}$, and above $1200^{\circ} \mathrm{C}$ its strength is comparable to or even weaker than that of tungsten carbide at room temperature. This thermomechanical behavior, quantified for the first time in controlled deformation experiments, provides both limitations and engineering guides for diamond applications in harsh and high-temperature conditions such as drilling, grinding, and high $P$-T laboratory experiments.

Crystal plasticity of diamond at high temperatures has been a subject of substantial interest. In natural diamonds, most of them are plastically deformed as manifested in the birefringence patterns ${ }^{20}$. Microstructure characterization and single-crystal $\mathrm{x}$-ray diffraction studies indicate that in addition to classic dislocation glide, mechanical twinning is another active mechanism underlying plastic deformation $^{21}$. Such micro twins have been observed around cobalt inclusions in synthetic diamonds ${ }^{22}$ and in naturally deformed pinkpurple diamonds from siberian kimberlites ${ }^{23}$. They were also reported for diamonds experimentally annealed at high pressure and temperature when embedded in hard silicates of olivine or garnet $^{19}$. In addition, such mechanical microtwins are common features in deformed materials with the diamond structure such as silicon ${ }^{24}$. It is, however, the first time that $\{111\}$ mechanical twinning is reported 
a)

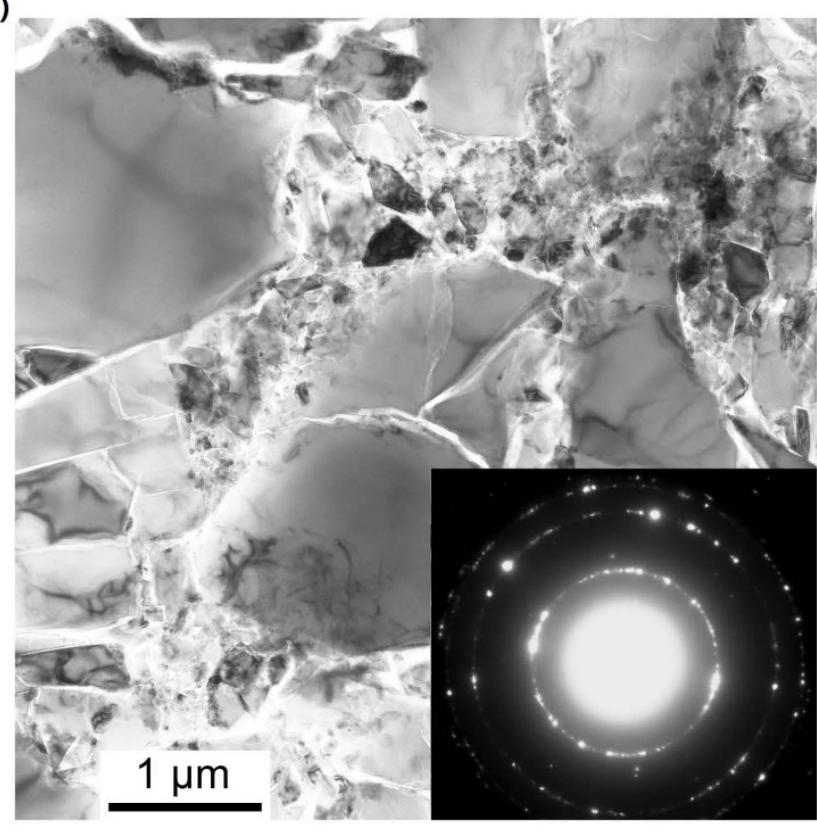

b)

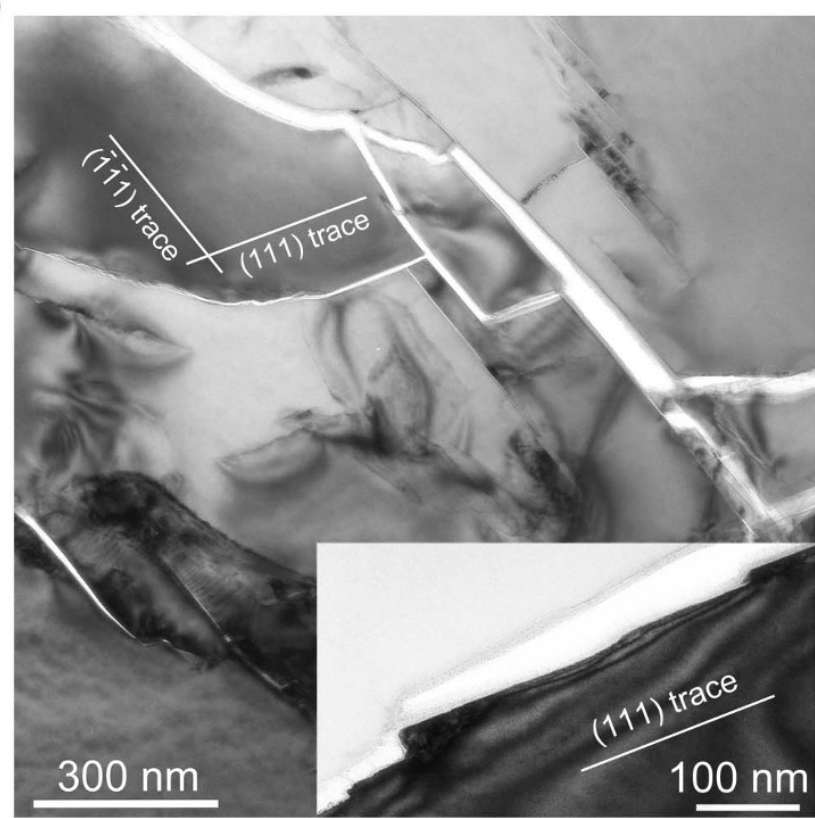

Figure 4 Bright-field (BF) TEM micrographs of the diamond sample recovered from room-temperature deformation. (a) Large grains almost free of dislocations are surrounded by small-grain areas; for smaller grains diffraction patterns exhibit spotty Debye rings. (b) BF TEM micrograph near $[1 \overline{1} 0]$ zone axis shows numerous $\{111\}$ cleavages cutting through a large broken grain. Note that the curved cracks consist of nano-steps along $\{111\}$ cleavages, as detailed in the inset.

as a major deformation mechanism in diamonds experimentally deformed at high temperatures. Last but not least, earlier reports from indentation experiments showed that diamonds with lower levels of nitrogen can more easily be deformed than diamonds with higher levels of nitrogen ${ }^{25}$. These observations indicate that nitrogen impurity may have profound effects on the deformation behavior of diamond crystals. Systematic studies of such effects under controlled deformation are thus warranted for a comprehensive understanding of crystal plasticity of diamond.

\section{Methods}

Uniaxial deformation experiments under confined high pressure. The polycrystalline diamond powder was purchased from Microdiamant. The aspurchased powder is more than $99.5 \%$ pure and has a grain size distribution of 4 to 6 microns as characterized by SEM. The uniaxial deformation experiments on polycrystalline diamond at high pressure-high temperature condition were performed at beamline X17B2 of the National Synchrotron Light Source (NSLS), Brookhaven National Laboratory, using a deformation-DIA (D-DIA ${ }^{15}$. The D-DIA apparatus is a modified version of the DIA ${ }^{26}$ that uses 6 hard anvils (primarily WC) to compress a sample assembly. For deformation under confined high pressure, the two vertical anvils can be driven independently to introduce compressive or tensile uniaxial straining. The pressure medium is a mixture of amorphous boron and epoxy resin for $\mathrm{x}$-ray transparency. The diamond powders were compacted in a boron nitride sleeve, which also isolates the sample from graphite furnace. Bulk differential stress was produced in the specimen with hard alumina pistons placed on both ends of the specimen. The sample was separated from the alumina pistons by nickel foils, which were used as strain markers for measuring the total strain.

In each of the experiments reported here, the pressure cell was first compressed hydrostatically to a desired pressure, followed by heating to the maximum temperature of $1200^{\circ} \mathrm{C}$ (the temperature was determined from previously established calibrations and the estimated errors are $\pm 50^{\circ} \mathrm{C}$ ). The sample was then deformed at a desired temperature by advancing top and bottom anvils, typically at the strain rates of $\sim 10^{-5} / \mathrm{s}$. During the experiment, a white X-ray beam of $50 \mu \mathrm{m} \times 50 \mu \mathrm{m}$ passed through the deformed sample in radial direction. With the use of a solid-state multielement detector that covers azimuth angles from $0^{\circ}-90^{\circ}$, diffraction data were collected periodically with $\sim 10$ minute counting time for each pattern. X-ray radiographs of the deformed column at corresponding deformation conditions were also taken by removing the collimation slits. This bulk information is used, as described next, to derive differential stress and strain for each experimental run. Note that the pressure was applied in this work because external pressure helps minimize flow of polycrystalline diamond along the radial direction when sample is under uniaxial loading. Within the stability filed of graphite pressure is also needed to stabilize diamond and hence to prevent partial graphitization of diamond under the present experimental temperatures. Our work, however, was not intended to systematically study the pressure effect on the deformation mechanisms of diamond.

The sample columns from X-ray radiographs are used to measure the changes in the sample length during the deformation experiment. Depending on the intensity contrast between sample and strain marker ( $\mathrm{Ni}$ foil), it is possible to measure the sample length changes within 1 microns. The total axial strain of the sample is calculated as: $\varepsilon=\left(l-l_{0}\right) / l_{0}$, where $l_{0}$ is the sample length at the onset of deformation and $l$ is the sample length under a given stress condition.

Derivation of microscopic strain and stress. Following the analytical method of ${ }^{27,28}$, the full-width-at-half-maximum of a diffraction peak, FWHM, is expressed in a length scale of $\AA(\Delta d)$, and the microscopic differential strain is calculated from Equation (1)

$$
\varepsilon_{\text {micro }}=\Delta d_{\varepsilon} / d=\sqrt{\Delta d_{\text {obs. }}^{2}-\Delta d_{\text {ins. }}^{2}} / d(P, T)
$$

where $\Delta d_{o b s}$ is the observed peak width under a given stress condition, $\Delta d_{i n s}$ is the peak width at a stress-free state, and $d$ is the d-spacing of a given lattice plane. In this work, the ambient diffraction data are chosen as a reference for zero strain. By multiplying the strain by an aggregate Young's modulus of $1050 \mathrm{GPa}$ for diamond, we can convert the strain to stress. Since this stress represents the local contact stress between crystal grains, it is conventionally termed microscopic stress.

Derivation of macroscopic strain and stress. Different from the microscopic stress which originates from the local grain-to-grain contact stress field, the macroscopic stress directly reflects the stress field applied to the entire sample, which can be quantified by measuring the peak position shift. The methods used here for macroscopic stress analysis are similar to those described by Uchida et al. ${ }^{29}$. We first determine lattice strain, $\varepsilon_{\text {macro }}(\varphi)=\left[\mathrm{d}_{0}(\varphi)-\mathrm{d}(\varphi)\right] / \mathrm{d}_{0}(\varphi)$, where $\varphi$ is the true azimuth angle, given by $\cos \varphi=\cos \theta \cos \chi(\theta$ and $\chi$ are the diffraction angle and detector azimuth), and $\mathrm{d}_{0}(\varphi)$ and $\mathrm{d}(\varphi)$ are $\mathrm{d}$-spacing values of a given lattice plane at the onset of deformation and at a certain stress state, respectively. The lattice strain, $\varepsilon_{\text {macro }}(\varphi)$, is fitted to the Equation (2) to obtain the differential lattice strain, $\varepsilon_{\text {macro, }}$.

$$
\varepsilon_{\text {macro }}(\varphi)=\varepsilon_{\text {macro }, p}-\varepsilon_{\text {macro,t }}\left(1-3 \cos ^{2} \varphi\right)
$$

With a cylindrical symmetry of the stress field in the D-DIA pressure cell the differential stress is defined by $t=\sigma_{1}-\sigma_{3}$, where $\sigma_{1}$ and $\sigma_{3}$ are the principal stresses in axial and radial directions, respectively. For a given lattice plane $h k l$, the differential strain can be converted into the differential stress by equation (3):

$$
t(h k l)=6 \varepsilon_{\text {macro,t }} \times G_{h k l}
$$

where $G_{h k l}$ is the shear's modulus for a lattice plane $h k l$, which can be derived from single-crystal elastic constants for diamond. Throughout this work, both the microscopic and macroscopic strains are derived from the lattice plane (111).

SEM and TEM characterizations. SEM characterizations were carried out using an FEI Inspect F SEM. For preparation of TEM thin sections, the cylindrical specimens were first embedded into epoxy and then cut perpendicularly to the compression axis 
a)

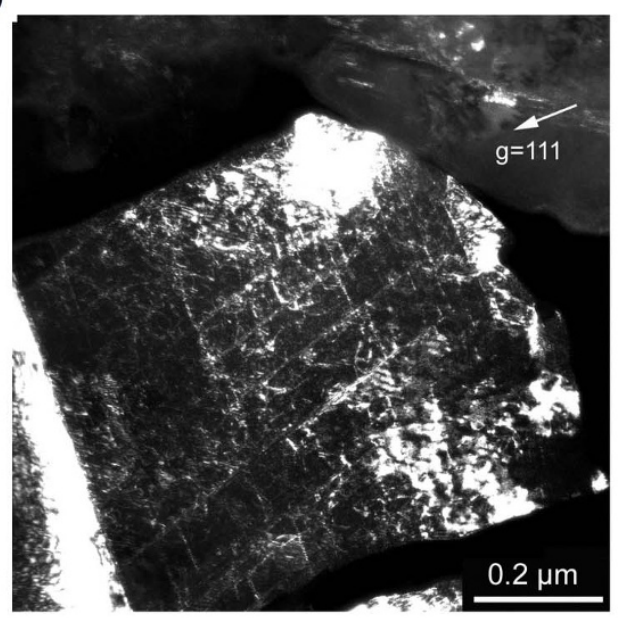

b)

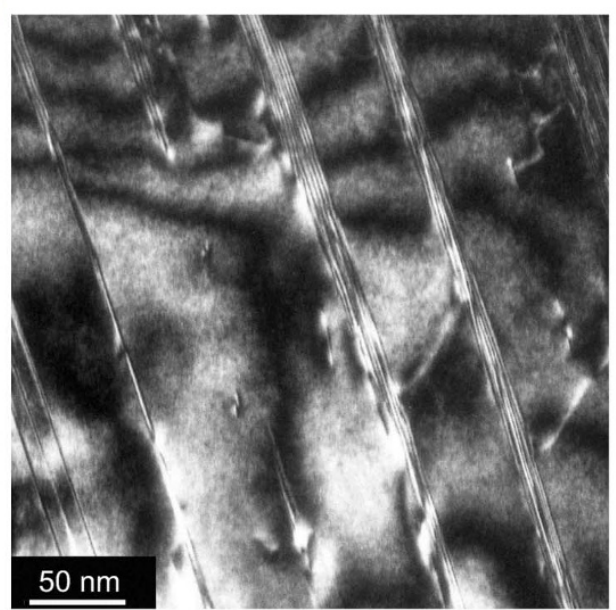

c)

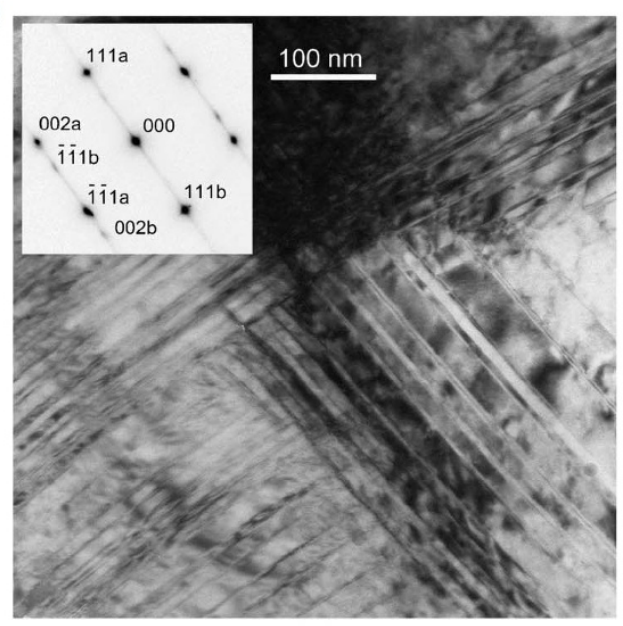

Figure $5 \mid$ TEM micrograph of the diamond sample recovered from the deformation experiment at $1200{ }^{\circ} \mathrm{C}$. (a) Weak-beam dark-field (WBDF) micrograph of a typical grain exhibiting numerous entangled dislocations and planar features (diffraction vector is indicated by $g$ ). (b) A DF micrograph showing planar deformation features. The observed moiré fringes are classically characteristic of stacking faults and microtwins; (c) $\mathrm{BF}$ micrographs of a grain exhibiting numerous $\{111\}$ microtwins. The inset shows the diffraction pattern of an area selected in the lower left corner of the image; splitting reflections and streaks are due to thin alternated twinned domains. and mechanically milled down to $\sim 25 \mu \mathrm{m}$ thickness. Prior to TEM investigation, the doubly-polished sections were examined in the optical microscope, ion-thinned to electron transparency (Ar beam accelerated at $5 \mathrm{kV}$ ), and coated with an amorphous carbon layer. A Philips CM30 electron microscope operating at $300691 \mathrm{kV}$ was used to investigate the specimens.

1. Field, J. E. The Properties of Natural and Synthetic Diamond. (Academic, London, 1997)

2. Telling, R. H., Pickard, C. J., Payne, M. C. \& Field, J. E. Theoretical Strength and Cleavage of Diamond. Phys. Rev. Lett. 84, 5160-5163 (2000).

3. Roundy, D. \& Cohen, M. L. Ideal strength of diamond, Si, and Ge. Phys. Rev. B 64, 212103 (2001).

4. Zhang, Y., Sun, H. \& Chen, C. Structural deformation, strength, and instability of cubic BN compared to diamond: A first-principles study. Phys. Rev. B 73, 144115 (2006).

5. Luo, X., Liu, Z., Xu, B., Yu, D., Tian, Y., Wang, H. \& He, J. Compressive Strength of Diamond from First-Principles Calculation. J. Phys. Chem. C 114, 17851-17853 (2010).

6. Pirouz, P., Cockayne, D. J. H., Sumida, N., Hirsch, P. \& Lang, A. R. Dissociation of Dislocations in Diamond. Proc. R. Soc., Lond. A 386, 241-249 (1983).

7. Yin, L., Yuan, Q., Li, M., Liu, Y., Xu, B. \& Hao, Z. Twin Diamond Crystals Grown at High Temperature and High Pressure from the Fe-Ni-C System. Chin. Phys. Lett. 19, 1371-1373 (2002).

8. Evens, T., Wild, R. K. \& Thomson, J. J. Plastic Deformation of Diamond at Temperatures below $1800^{\circ} \mathrm{C}$. Philosophical Magazine 13, 209-210 (1965).

9. Field, J. E. \& Pickles, C. S. J. Strength, fracture and friction properties of diamond Diam. Relat. Mater. 5, 625-634 (1996).

10. Mao, H. K. \& Hemley, R. J. Optical transitions in diamond at ultrahigh pressures. Nature 351, 721-724 (1991).

11. Eremets, M. I., Trojan, I. A., Gwaze, P., Huth, J., Boehler, R. \& Blank, V. D. The strength of diamond. Appl. Phys. Lett. 87, 141902 (2005).

12. Akahama, Y. \& Kawamura, H. Raman study on the stress state of [111] diamond anvils at multimegabar pressure. J. Appl. Phys. 98, 083523-083526 (2005).

13. Hemley, R. J., Mao, H. K., Shen, G., Badro, J., Gillet, P., Hanfland, M. \& Hausermann, D. X-ray Imaging of Stress and Strain of Diamond, Iron, and Tungsten at Megabar Pressures. Science 276, 1242-1245 (1997).

14. Weidner, D. J., Wang, Y. \& Vaughan, M. T. Strength of Diamond. Science 266, 419-422 (1994).

15. Wang, Y., Durham, W. B., Getting, I. C. \& Weidner, D. J. The deformation-DIA: A new apparatus for high temperature triaxial deformation to pressures up to 15 GPa. Rev. Sci. Instrum. 74, 3002-3009 (2003).

16. Novikov, N. V. \& Dub, S. N. Fracture toughness of diamond single crystals. J. Hard Mater. 2, 3-11 (1991).

17. Humble, P. \& Hinnink, R. H. J. Plastic deformation of diamond at room temperature. Nature 273, 37-39 (1978).

18. Nistor, L., Teoderescu, V., Landuyt, J. V. \& Ralchenko, V. The origin of anomalous spots in the electron diffraction patterns of diamond films. Proceeding from the European Congress on Electron Microscopy EUREM XII, Brno, Czech Republic, July 9-14, p. 495 (2000).

19. Shyryaev, A. A., Frost, D. J. \& Langenhorst, F. Impurity diffusion and microstructure in diamonds deformed at high pressures and temperatures. Diam. Relat. Mater. 16, 503-511 (2007).

20. Lang, A. R. Causes of Birefringence in Diamond. Nature 213, 248-251 (1965).

21. Titkov, S. V., Krovoviche, S. V. \& Organova, N. I. Plastic deformation of natural diamonds by twinning: evidence from X-ray diffraction studies. Mineral. Mag. 76, 143-149 (2012).

22. Langenhorst, F., Poirier, J. P. \& Frost, D. J. TEM observations of microscopic inclusions in synthetic diamond. J. Mater. Sci. 39, 1865-1867 (2004).

23. Mineeva, R. M., Speransky, A. V., Titkov, S. V. \& Zudin, N. G. The ordered creation of paramagnetic defects at plastic deformation of natural diamonds. Phys. Chem. Miner. 34, 53-58 (2007).

24. Houben, L., Luysberg, M. \& Carius, R. Microtwinning in microcrystalline silicon and its effect on grain-size measurements. Phys. Rev. B 67, 045312-045321 (2003)

25. Brookes, E. J., Comins, J. D., Daniel, R. D. \& Erasmus, R. M. A study of plastic deformation profiles of impressions in diamond. Diam. Relat. Mater. 9 1115-1119 (2000).

26. Osugi, J., Shimizu, K., Inoue, T. \& Yasunami, K. A Compact Cubic Anvil High Pressure Apparatus. Rev. Phys. Chem. Jpn 34, 1-6 (1964).

27. Zhao, Y. \& Zhang, J. Microstrain and grain-size analysis from diffraction peak width and graphical derivation of high-pressure thermomechanics. J. Appl. Crystallog. 41, 1095-1108 (2008).

28. Yu, X., Zhang, J., Wang, L., Ding, Z., Jin, C. \& Zhao, Y. Comparative studies of constitutive properties of nanocrystalline and bulk iron during compressive deformation. Acta Mater. 59, 3384-3389 (2011).

29. Uchida, T., Wang, Y., Rivers, M. L. \& Sutton, S. R. Earth Planet. Sci. Lett. 226, 117-126 (2004)

\section{Acknowledgment}

This research is conducted at Los Alamos National Laboratory, which is operated by Los Alamos National Security LLC under DOE Contract DE-AC52-06NA25396, with the 
supports from DOE/EERE-ITP nanoManufacturing program. The UNLV High Pressure Science and Engineering Center (HiPSEC) is a DOE NNSA Center of Excellence supported by Cooperative Agreement DE-FC52-06NA27684, The deformation experiments were carried out at beam line X17B2 at the National Synchrotron Light Source, Brookhaven National Laboratory. Use of X17B2 beamline was supported by COMPRES, the

Consortium for Materials Properties Research in Earth Sciences, under NSF Cooperative Agreement EAR 01-35554. The TEM national facility in Lille (France) is supported by the Conseil Regional du Nord-Pas de Calais, the European Regional Development Fund (ERDF), and the Institut National des Sciences de l'Univers (INSU, CNRS).

\section{Author contributions}

XY, LW, and JZ performed the experiments; PR carried out TEM characterization and image interpretation; ZL performed SEM characterization; XY analyzed the data and drafted the manuscript; JZ and YZ edited the manuscript and also provided inputs for data interpretation. All authors reviewed the manuscript.

\section{Additional information}

Competing financial interests: The authors declare no competing financial interests. License: This work is licensed under a Creative Commons Attribution-NonCommercial-NoDerivs 3.0 Unported License. To view a copy of this license, visit http://creativecommons.org/licenses/by-nc-nd/3.0/.

How to cite this article: Yu, X. et al. Constitutive Law and Flow Mechanism in Diamond Deformation. Sci. Rep. 2, 876; DOI:10.1038/srep00876 (2012). 